\title{
A FINITENESS THEOREM FOR LINEAR SEMIGROUPS
}

\author{
YECHEZKEL ZALCSTEIN
}

(Communicated by Bhama Srinivasan)

ABSTRACT. A classical theorem of Burnside on the finiteness of periodic linear groups is generalized to semigroups.

The following theorems are classical.

BURNSIDE'S THEOREM [3, (36.1)]. A periodic group of matrices over a field of characteristic 0 of bounded period is finite.

SCHUR'S THEOREM [3, (36.2)]. A finitely generated periodic group of matrices over a field is finite.

Schur's theorem has been generalized to semigroups in [5]. Burnside's theorem, however, is false for semigroups without further restrictions. In [7], Burnside's theorem was generalized to inverse semigroups. In this note we generalize the theorem further to a wider class of semigroups. The theorem is of interest since all the finiteness conditions in the literature with the sole exception of Theorem 8 of [4] assume the semigroups are finitely generated.

We assume familiarity with the elementary representation theory of semigroups (e.g., Chapter 5 of [2]). All undefined terminology and notation follows [2].

Recall that a class of representations is called complete if the direct sum of the representations is faithful on the semigroup algebra $K[S]$.

THEOREM. Let $S$ be a periodic semigroup of matrices over a field $K$ of characteristic 0 of bounded period. If

(1) $S$ has a complete set of irreducible representations then $S$ is finite.

PROOF. By the remarks following the proof of the theorem of [6], $S$ satisfies $M_{J}$, the d.c.c. on principal two-sided ideals, thus all its principal factors are completely (0)-simple or nilpotent. Let $J$ be a regular $J$-class of $S$. Then $J^{0}$ is isomorphic to a Rees matrix semigroup $M^{0}(G ; I, \Lambda ; P)$. Further, $G$ is finite by Burnside's theorem. Hence $J^{0}$ has a finite number of inequivalent irreducible representations. Since $S$ satisfies $M_{J}$, it follows from the Munn-Ponizovskii theorem (5.33 in [2]) that every irreducible representation of $S$ is an extension of an irreducible representation of a regular $J$-class. Again by [6], $S$ has finitely many regular $J$-classes. Thus $S$ has

Received by the editors May 8, 1987.

1980 Mathematics Subject Classification (1985 Revision). Primary 20M30; Secondary 20M20, $20 \mathrm{M} 25$.

Research supported, in part, by NSF grant DCR 8602319 while the author was at Memphis State University. Any opinions, findings, and conclusions or recommendations expressed in this publication are those of the author and do not necessarily reflect the views of the National Science Foundation. 
only finitely many irreducible representations. If $h$ is an irreducible representation of $S$, then $h(S)$ can be shown to be finite by the argument used in the proof of Burnside's theorem in [3]. By (1) $S$ has a complete set of irreducible representations and since by the above argument, there are only finitely many, their direct sum is faithful on the semigroup algebra $K[S]$. Thus $K[S]$ is finite dimensional and $S$ must be finite.

The hypothesis (1) may seem excessively restrictive. However, the theorem is best possible in the following sense. For groups, by [1], (1), together with existence of a uniform upper bound on the degrees of the representations is equivalent to

$\left(1^{\prime}\right)$ The semigroup algebra of $S$ satisfies a polynomial identity over any field of characteristic 0 .

However, the semigroup $S$ of all strictly lower triangular matrices over an infinite field of characteristic 0 is periodic of bounded period, satisfies $\left(1^{\prime}\right)$ and also $M_{J}$, yet is infinite.

\section{REFERENCES}

1. S. A. Amitsur, Groups with representations of bounded degree. II, Illinois J. Math. 5 (1961), 198-205.

2. A. H. Clifford and G. B. Preston, The algebraic theory of semigroups, Math. Surveys Mongr., no. 7, Amer. Math. Soc., Providence, R. I., 1961.

3. C. W. Curtis and I. Reiner, Representation theory of finite groups and associative algebras, Interscience, New York, 1962.

4. E. Hotzel, On finiteness conditions in semigroups, J. Algebra 60 (1979), 352-370.

5. R. McNaughton and Y. Zalcstein, The Burnside problem for semigroups, J. Algebra 34 (1975), 292-299.

6. J. Okninski, $\pi$-regular matrix semigroups, Proc. Amer. Math. Soc. 93 (1985), 215-217.

7. Y. Zalcstein, Finiteness conditions for matrix semigroups, Proc. Amer. Math. Soc. 38 (1973), 247-249.

Division of Computer and Computation Research, National SCience FounDATION, WASHINGTON, D. C. 20550 